ИЗВЕСТИЯ АКАДЕМИИ НАУК ЭСТОНСКОИ ССР. ФИЗИКА * МАТЕМАТИКА

PROCEEDINGS OF THE ACADEMY OF SCIENCES OF THE ESTONIAN SSR.

PHYSICS * MATHEMATICS

$1987,36,4$

удК $535.37 ; 548.736$

Светлана ЗАЗУБОВИЧ, В. НАГИРНЫИ, Т. СООВИК

\title{
НОВАЯ НЕЦЕНТРАЛЬНАЯ СИСТЕМА - РТУТЕПОДОБНЫЕ ИОНЫ В ЩЕЛОЧНОГАЛОИДНЫХ КРИСТАЛЛАХ
}

\author{
(Представил Ч. Лущик)
}

Исследованы особенности низкотемпературной кинетики затухания триплетной люминесценции $\mathrm{In}^{+}$-центров в щелочногалондных кристаллах (ЩГК) и влияние на нее электрических полей. Показано, что ион $\mathrm{In}^{+}$в галогенидах калия и рубидия в релаксированном возбужденном состоянии (PBC) смещен из узла решетки в направлении, перпендикулярном оси ян-теллеровского искажения. Туннельые переходы между разными нецентральными положениями иона $\mathrm{In}^{+}$приводят к туннельному расщеплению тетрагональных метастабильных минимумов адиабатического потенциала триплетного PBC. С радиационным распадом туннельных уровней связаны наблюдающиеся при низких температурах два медленных компонента затухания $A_{T}$-излучения этих систем. На основании полученных данных интерпретирована обнаруженная ранее методом оптически детектируемого электронного парамагнитного резонанса (ОД ӘПР) орторомбическая структура PBC $\mathrm{Ga}^{+}$-центров в ЩГК.

\section{1. Введение}

В [ $\left.{ }^{1}\right]$ нами было проведено систематическое исследование кинетических характеристик триплетной люминесценции $\mathrm{In}^{+}$-центров в семи ЩГК, различающихся катионом или анионом основания. При низких температурах в затухании $A_{T}$-излучения $\mathrm{In}^{+}$-центров в галогенидах калия и рубидия были обнаружены два медленных компонента - $M K$ и $M K^{\prime}$ (см. также $\left.\left[{ }^{2}\right]\right)$ и изучены их спектральные и поляризационные характеристики. Наблюдаемые эффекты были интерпретированы как первое проявление нецентрального (НЦ) положения ртутеподобного иона в РВС в решетке ЩГК.

Настоящая работа посвящена дальнейшему детальному исследованию низкотемпературной кинетики затухания $A_{T}$-излучения $\mathrm{In}^{+}$-центров и влияния на нее электрических полей.

\section{2. Кинетика затухания излучения}

\section{1. Экспериментальные результаты}

Исследуемую область температур можно условно разделить на четыре характерных участка (рис. 1).

При $\boldsymbol{T}<\boldsymbol{T}_{1}$ времена затухания $\tau_{M K}$ и $\tau_{M K^{\prime}}$ почти не зависят от температуры. Отношение $\tau_{M K} / \tau_{M K^{\prime}}$ растет в ряду анионов $\mathrm{Cl}^{-} \rightarrow \mathrm{Br}^{-} \rightarrow \mathrm{I}^{-}$и катионов $\mathrm{K}^{+} \rightarrow \mathrm{Rb}^{+}$, т. е. с увеличением постоянной решетки. Соотношение светосумм $S_{M K^{\prime}} / S_{M K}$ тоже практически не изменяется с температурой, но в существенной степени зависит от аниона основания и растет в ряду $\mathrm{Cl}^{-} \rightarrow \mathrm{Br}^{-} \rightarrow \mathrm{I}^{-}$(см. подробнее в [ $\left.\left.{ }^{1}\right]\right)$.

В интервале температур $T_{1}-T_{2} \tau_{M K}$ заметно укорачивается, $\tau_{M K^{\prime}}$ тоже становится короче, а соотношение светосумм $S_{M K^{\prime}} / S_{M K}$ резко уменьшается. При $T=T_{2}$ более быстрый из двух компонентов $\left(M K^{\prime}\right)$ 


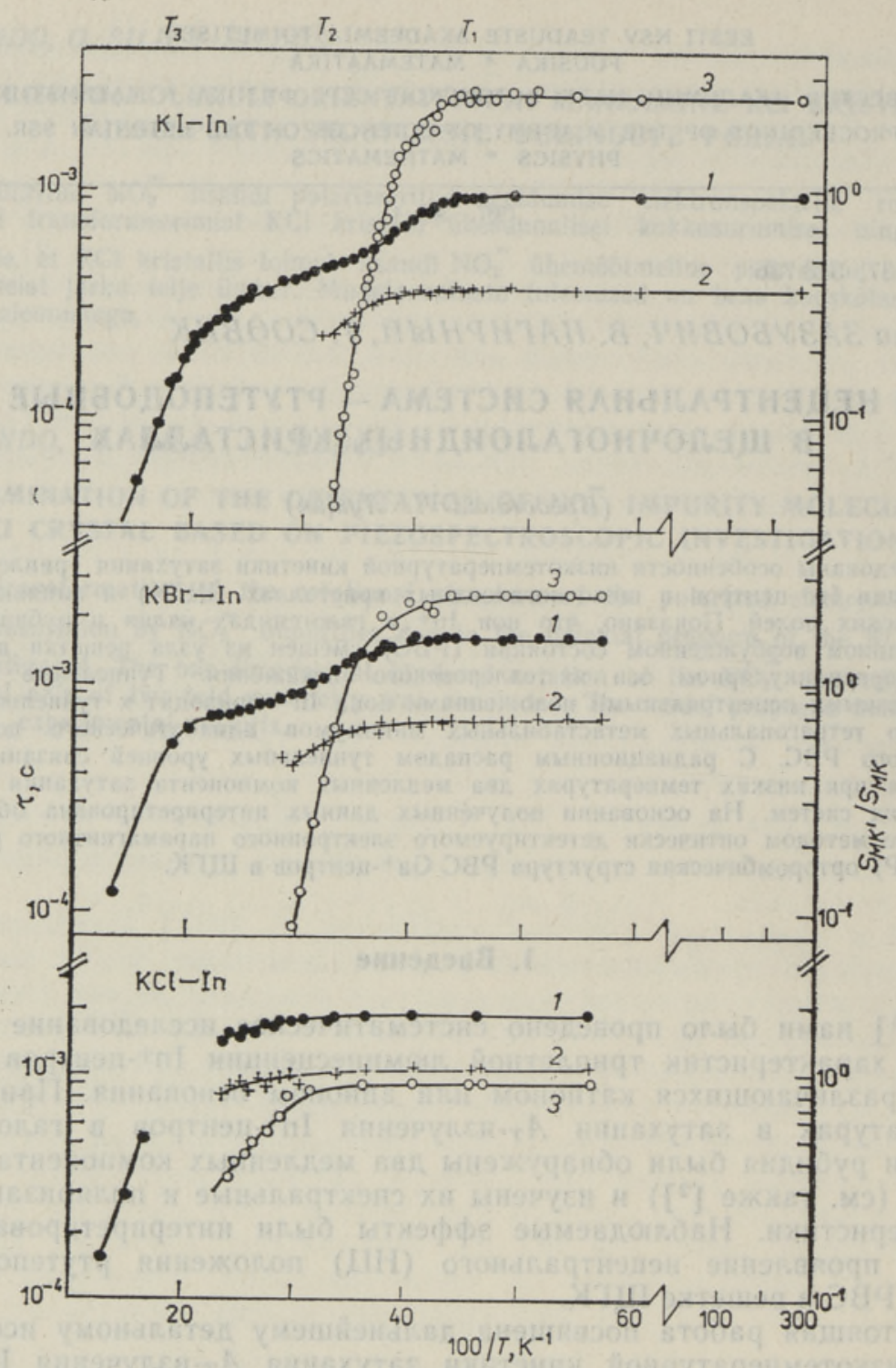

Рис. 1. Температурные зависимости времен затухания $\tau_{M K}(1)$ и $\tau_{M K^{\prime}}$ (2) и соотношения светосумм $S_{\mathbf{M ~ K}^{\prime}} / S_{M K}$ (3) двух медленных компонентов затухания $A_{T}$-излучения $\mathrm{In}^{+}$-центров в $\mathrm{KI}-\mathrm{In}, \mathrm{KBr}-\mathrm{In}$ и $\mathrm{KCl}-\mathrm{In}$.

пропадает. В ряду анионов $\mathrm{Cl}^{-} \rightarrow \mathrm{Br}^{-} \rightarrow \mathrm{I}^{-}$значения $T_{1}$ и $T_{2}$ смещаются в сторону более низких температур.

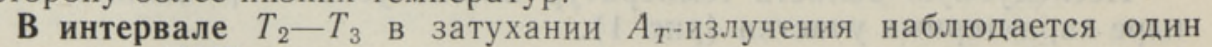
компонент $(M K)$, время затухания которого сравнительно мало меняется с температурой.

Резкое укорачивание $\tau_{M K}$ при $\boldsymbol{T}>\boldsymbol{T}_{3}$ обусловлено хорошо известным процессом - термическим заселением излучательных минимумов триплетного состояния. Зависимости $\tau_{M K}\left(T^{-1}\right)$ в этой области близки для систем с одинаковыми анионами. Это говорит о том, что параметры триплетного РВС (вероятности излучательных и безызлучательных переходов, спиновое расщепление) определяются в существенной степени галоидным окружением примеси. 


\section{2. Обсуждение результатов}

Описанные экспериментальные факты (см. также [1) мы объясняем тем, что в галогенидах калия и рубидия ион In+ в PBC при низких температурах смещен из узла решетки. Как будет показано ниже, это смещение происходит в одном из четырех направлений оси $C_{4}$ кристалла в плоскости, перпендикулярной оси ян-теллеровского искажения (подробнее см. раздел 3). Эти НЦ-положения разделены энергетическим барьером $V_{0}$, величина которого растет в ряду анионов $\mathrm{Cl}^{-} \rightarrow \mathrm{Br}^{-} \rightarrow \mathrm{I}^{-}$и катионов $\mathrm{K}^{+} \rightarrow \mathrm{Rb}^{+}$(ср. с $\left.\left[{ }^{3,4}\right]\right)$. Вследствие переходов между НЦ-положениями четырехкратное ориентационное вырождение адиабатического потенциала частично снимается, и метастабильный уровень $\mathrm{PBC} \mathrm{In}^{+}$-центров расщепляется на три туннельных уровня $\left(A, E\right.$ и $B$ в порядке возрастания их энергии $\left.\left[{ }^{5}\right]\right)$. С радиационным распадом двух нижних из них $(A$ и $E)$ мы связываем два медленных компонента $\left(M K\right.$ и $\left.M K^{\prime}\right)$ затухания исследуемого излучения *. Чем меньше барьер $V_{0}$, тем больше скорость туннельных переходов, а отсюда тем больше и величина туннельного расщепления $\Delta\left[{ }^{6}\right]$. Следовательно, $\Delta$ должно расти в ряду $\mathrm{KI} \rightarrow \mathrm{KBr} \rightarrow \mathrm{KCl}$.

Причиной НЦ-смещения иона $\mathrm{In}^{+}$в PBC может являться смешивание

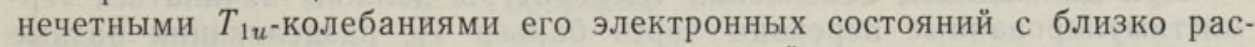
положенными состояниями противоположной четности квазимолекулы, включающей ион примеси и его ближайшее окружение (псевдоэффект Яна-Теллера) или квадратичное взаимодействие с $T_{1 u}$-колебаниями (см. обзор $\left.\left[{ }^{7}\right]\right)$.

Постоянство времен затухания $\tau_{M K}$ и $\tau_{M K^{\prime}}$ и соотношения $S_{M K^{\prime}} / S_{M K}$ в области температур $T<T_{1}$ говорят о том, что термостимулированные переходы между туннельными состояниями при этих температурах не происходят. Следовательно, вероятность радиационного распада нижайшего состояния $(A)$ определяется как $\gamma_{1}(A)=\tau_{M K}{ }^{-1}$. В этих условиях наблюдается явная корреляция времени затухания более медленного компонента $\left(\tau_{M K}\right)$ с глубиной НЦ-минимумов: чем они глубже, тем меньше вероятность их радиационного распада. Время затухания $\tau_{M K^{\prime}}$ при $T<T_{1}$ определяется вероятностью радиационного распада Е-состояния $\gamma_{1}(E)$ и вероятностью $\left(p_{0}\right)$ однофононных спонтанных безызлучательных переходов из $E$ - в $A$-состояние: $\tau_{M K^{\prime}}^{-1}=\gamma_{1}(E)+p_{0}$.

Как было показано в $\left[{ }^{2}\right]$, радиационный распад метастабильных минимумов $\mathrm{In}^{+}$-центров обусловлен взаимодействием с $T_{2 g}$-колебаниями и сверхтонким взаимодействием, подмешивающими излучательные состояния триплетного РВС к метастабильным [ $\left.{ }^{8}\right]$. В связи с этим вероятности $\gamma_{1}(E)$ и $\gamma_{1}(A)$ могут различаться из-за разного расстояния туннельных уровней $E$ и $A$ от излучательного состояния. Различие $\gamma_{1}(E)$ и $\gamma_{1}(A)$ может быть обусловлено также тем, что нечетными колебаниями по-разному подмешиваются состояния противоположной четности к $E$ - и $A$-состояниям. Кроме того, $\tau_{M K^{\prime}}<\tau_{M K}$ и из-за безызлучательных переходов $E \rightarrow A$. Сама возможность наблюдения проявлений туннельного расщепления в кинетике затухания излучения, а также отсутствие температурной зависимости $\tau_{M K}$ и $\tau_{M K^{\prime}}$ при $T<T_{1}$ свидетельствует о том, что скорость туннельных переходов между различными НЦ-положениями значительно превосходит вероятность излучательного распада метастабильных минимумов.

\footnotetext{
* Вероятность радиационного распада верхнего туннельного $B$-состояния гораздо меньше, чем $A$ - и $E$-состояний [5], поэтому в затухании излучения при низких температурах наблюдаются лишь два компонента. Наличне этого состояния должно оказывать влияние на кинетику затухания излучения при температурах, когда становятся возможными переходы между $B$-, $E$ - и $A$-уровнями,
} 
Если предположить, что оптически первоначально заселяется верхний $(E)$ уровень, то соотношение $S_{M K^{\prime}} / S_{M K}$ при $T<T_{1}$ определяется отношением вероятности безызлучательных переходов $(p)$ из верхнего в нижнее туннельное состояние к вероятности радиационного распада верхнего состояния. Тогда тот факт, что в KI-In соотношение $S_{M K^{\prime}} / S_{M K}$ значительно больше, чем у $\mathrm{KCI}$-In, говорит о том, что $p$ уменьшается с увеличением НЦ-смещения.

При $T>T_{1}$ становятся возможными термостимулированные переходы $A \rightarrow E$ с вероятностью $p \operatorname{exp~}(-\Delta / k T)$, приводящие к укорачиванию $\tau_{M K}$ и $\tau_{M K^{\prime}}$ и к резкому перераспределению светосумм $S_{M K}$ и $S_{M K^{\prime}}$. Из температурной зависимости $\tau_{M K}$ можно оценить величину туннельного расщепления $\Delta$. В KI-In она составляет $(7 \pm 1) \times 10^{-4}$ эВ и растет в ряду $\mathrm{KI} \rightarrow \mathrm{KBr} \rightarrow \mathrm{KCl}$, о чем свидетельствует смещение в этом ряду области $T_{1}-T_{2}$ (рис. 1), в которой происходит перераспределение светосумм $S_{M K}$ и $S_{M K^{\prime}}$, в сторону более высоких температур.

При $T \geqslant T_{2}$, когда $k T \gg \Delta$, более быстрый компонент $\left(M K^{\prime}\right)$ исчезает и в затухании $A_{T}$-излучения наблюдается только один равновесный компонент. В этой области температур, однако, НЦ-смещение иона примеси еще может сохраняться.

При дальнейшем повышении температуры должны начинаться термостимулированные переходы через барьер $V_{0}$, разделяющий разные НЦ-минимумы. Это соответствует переходу иона примеси в центральносимметричное положение. Чем глубже НЦ-минимумы, тем при более высокой температуре исчезает нецентральность. Однако, чем больше $V_{0}$, тем меньше $\Delta$, т. е. тем при более низкой температуре исчезает $M K^{\prime}$. При очень большом $V_{10}$ даже при предельно низких температуpax $\Delta \ll k T$. В этом случае НЦ-положение иона примеси не проявится в кинетике затухания излучения. Весьма вероятно, что такая ситуация реализуется в случае $\mathrm{Sn}^{2+}$ - и $\mathrm{Ge}^{2+}$-центров.

Из рис. 1 видно, что в $\mathrm{KCl}$ - In два медленных компонента затухания, а следовательно, и НЦ-смещение $\mathrm{In}^{+}$, сохраняется до $4 \mathrm{~K}$. Так как барьер $V_{0}$ в KI-In должен быть больше, чем в $\mathrm{KCl}$ - In, то термические переходы в излучательное состояние в KI-In, возможно, начинаются еще до того, как исчезает нецентральность.

Как отмечалось в [ $\left.{ }^{1}\right]$, мы связываем наблюдаемую методом ОД ЭПР орторомбическую симметрию РВС Ga+-центров с НЦ-положением иона $\mathrm{Ga}^{+}$. Исчезновение орторомбического искажения $T$-минимумов PBC (или связанного с ним расщепления излучательного дублета) могло бы быть индикатором перехода иона примеси в центральносимметричное положение. Наши предварительные исследования кинетики затухания $A_{T}$-излучения $\mathrm{Ga}^{+}$-центров в $\mathrm{KCl}-\mathrm{Ga}$ показали, что $M K^{\prime}$ исчезает уже к $1 \mathrm{~K}$, однако, судя по спектрам ОД ЭПР $[9,10]$, орторомбическая симметрия PBC (а значит, и НЦ-смещение иона $\mathrm{Ga}^{+}$) coхраняется по крайней мере до $2 \mathrm{~K}$. Очевидно, при повышении температуры она должна исчезнуть. K сожалению, температурные зависимости спектров ОД ЭПР $\mathrm{Ga}^{+}$-центров в ЩГК пока не исследованы.

\section{3. Влияние электрического поля на кинетику затухания $A_{T}$-излучения $\mathrm{In}^{+}$-центров}

Эксперименты в электрическом поле проводились нами главным образом для определения направления смещения иона $\mathrm{In}^{+}$из узла решетки, в частности для выяснения, происходит это смещение вдоль оси ян-теллеровского искажения или перпендикулярно ей. Известно, что в электрическом поле туннельные уровни НЦ-системы претерпевают дополнительное расщепление, если поле приложено в направлении, в 
котором возможно НЦ-смещение иона примеси. В случае, если НЦ-смещение наблюдается в возбужденном состоянии, изменения в процессах релаксации, вызванные этим расщеплением, могут проявиться в кинетике затухания излучения. В нашем случае изменений в кинетике затухания излучения можно ожидать, приложив электрическое поле вдоль оси ян-теллеровского искажения центра, если ион $\mathrm{In}^{+}$смещается по этой оси, или перпендикулярно ей, если смещение иона In+ происходит в плоскости, перпендикулярной оси ян-теллеровского искажения.

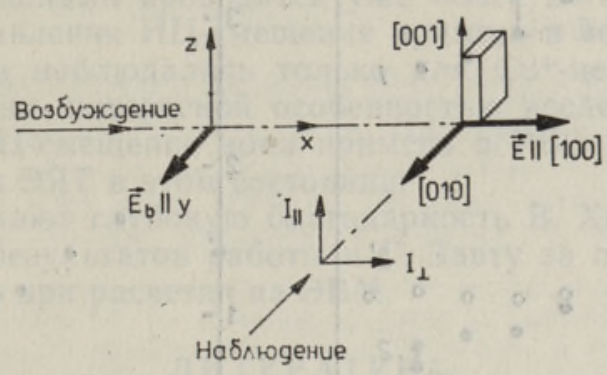

Рис. 2. Условия возбуждения и наблюдения $A_{T}$-люминесценции $\mathrm{In}^{+}$-центров в электрическом поле.

Условия возбуждения и наблюдения люминесценции в электрическом поле были выбраны так, чтобы четко разграничить экспериментально эти два случая (рис. 2). При возбуждении In+-центра светом, поляризованным вдоль оси $y$, преимущественно заселяются $X_{x^{-}}$и $Z_{z}$-метастабильные минимумы триплетного возбужденного состояния. В $X_{x}$-минимуме ось ян-теллеровского искажения направлена по $x$, a направление излучательных переходов - вдоль $y$ и $z$. В $Z_{z}$-минимуме ось ян-теллеровского искажения направлена по $z$, а направление излучательных переходов - вдоль $x$ и $y$. Если электрическое поле $\vec{E} \| x$, а $I_{\|} \| z$ и $I_{\perp} \| x$, то влияние электрического поля на кинетику затухания излучения обнаружится в $I_{\|}$, если смещение иона In+ происходит вдаль оси ян-теллеровского искажения, и в $I_{\perp}$, если это смещение происходит перпендикулярно оси.

Исследования показали, что электрическое поле (напряженностью до 6 кВ/мм) практически не влияет на $I_{\|}$. При измерении временной зависимости $I_{\perp}$, однако в KI-In наблюдалось некоторое изменение $\tau_{M K}, \tau_{M K^{\prime}}$ и соотношения $S_{M K^{\prime}} / S_{M K}$ с ростом $\vec{E}$ (рис. 3 ), указывающее на то, что ион $\mathrm{In}^{+}$в РВС смещается в плоскости, перпендикулярной оси ян-теллеровского искажения. Проведенный нами анализ спектров ОД ЭПР $\mathrm{Ga}^{+}$-центров, измеренных в $\left[{ }^{10}\right]$, показал, что в $\mathrm{PBC}$ ион $\mathrm{Ga}^{+}$ также смещается в одном из направлений $\langle 100\rangle$, перпендикулярном оси ян-теллеровского искажения. Полагая, что наблюдаемые явления в случае $\mathrm{In}^{+}-$и $\mathrm{Ga}^{+}$-центров имеют одинаковую природу, мы предполагаем аналогичное смещение и для иона $\mathrm{In}^{+} * *$.

** В этом случае каждый уровень ртутеподобного иона в РВС четырежды вырожден. Туннельные переходы приводят к расщеплению метастабильного состояния на туннельные $A-, E$ - и $B$-уровни [5]. В электрическом поле $E \|[100] E$-состояние расщепляется на два $B$-состояния: в общем случае меняются и энергии состояний. 

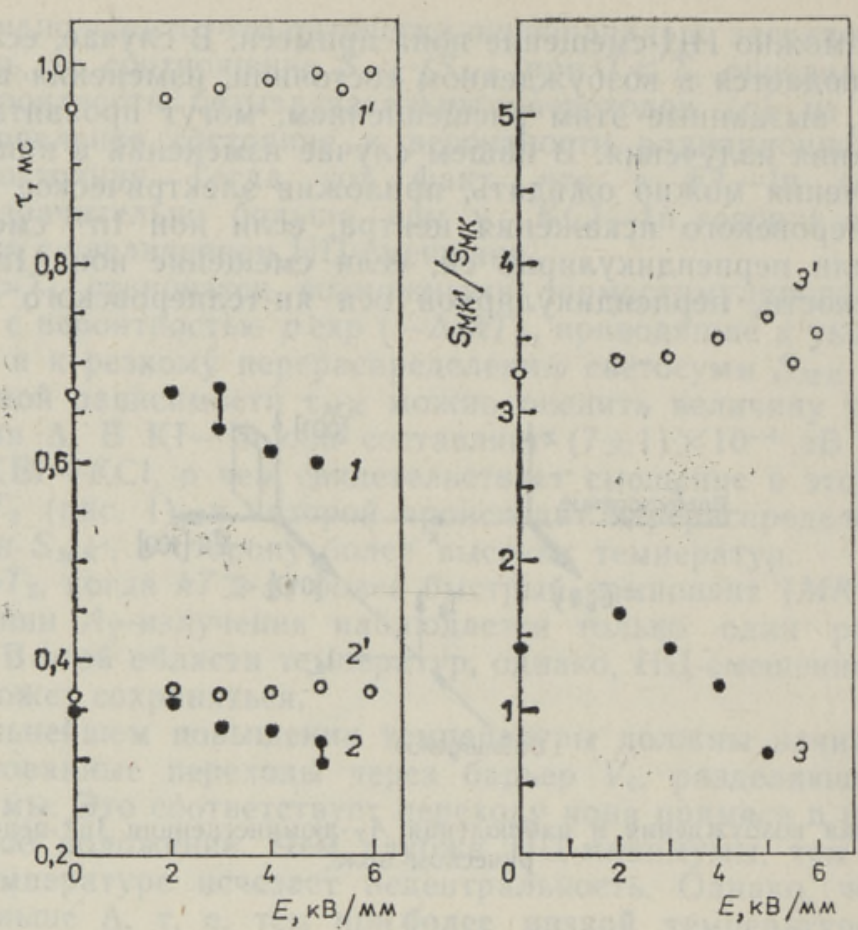

Рис. 3. Зависимости времен затухания $\tau_{M \boldsymbol{K}}\left(I, I^{\prime}\right)$ и $\tau_{M K^{\prime}}\left(2,2^{\prime}\right)$ и соотношения светосумм $S_{M K^{\prime}} / S_{M K}\left(3,3^{\prime}\right)$ двух медленных компонентов затухания $A_{T}$-излучения KI-In от напряженности электрического поля $\vec{E} \|[100]$ при $2,6 \mathrm{~K}(1-3)$ и $1,85 \mathrm{~K}$ $\left(1^{\prime}-3^{\prime}\right)$. Измерено в условиях рис. 2 для $I_{\perp}$.

Такое смещение ионов примеси в РВС, возможно, связано как с сильной анизотропией волновой p-функции, вследствие чего эффективный ионный радиус примеси минимален в плоскости, перпендикулярной направлению ян-теллеровского искажения, так и с низкой симметрией ближайшего окружения иона примеси вследствие статического эффекта Яна-Теллера (ЭЯТ).

Действие электрического поля на исследуемые системы весьма многогранно, поэтому можно дать лишь качественное объяснение наблюдаемым зависимостям $\tau(\vec{E})$ и $S_{M K^{\prime}} / S_{M K}(\vec{E})$. Известно, что электрическое поле приводит, в частности, к увеличению скорости релаксации $\left[{ }^{11}\right]$, т. е. к увеличению вероятности безызлучательных переходов между туннельными уровнями $(p)$. Однако увеличение глубины НЦминимумов (из-за понижения энергии $A$-состояния в электрическом поле) приводит, как уже отмечалось, к уменьшению $p$ и $\gamma_{1}$. В электрическом поле $p$ и $\gamma_{1}$ могут изменяться и вследствие изменения симметрии состояний. В связи с этим в разных температурных областях действие поля проявляется по-разному.

При $T<T_{1}$, возможно, из-за понижения энергии $A$-состояния в поле уменьшаются $\gamma_{1}$ и $p$, а следовательно, растут $\tau_{M K}, \tau_{M K^{\prime}}$ и $S_{M K^{\prime}} / S_{M K}$ (рис. 3, кружки). При $T>T_{1}$ эффекты, обусловленные изменением $(\Delta E)$ энергий туннельных уровней в электрическом поле, выражены слабее (т. к. $\Delta E \ll \kappa T)$, и $\tau_{M K}, \tau_{M \kappa^{\prime}}$ и $S_{M K^{\prime}} / S_{M K}$ уменьшаются с ростом $\vec{E}$ вследствие увеличения вероятности безызлучательных переходов $p$ (рис. 3 , точки). 
Сравнительно слабое влияние электрического поля на кинетику затухания исследуемого излучения объясняется тем, что туннельное расщепление $\Delta$ у In+-центров велико и даже в максимальных использованных нами полях $\left(6 \times 10^{4} \mathrm{~B} / \mathrm{cm}\right) \Delta \geqslant p_{\text {нц }} \vec{E}\left(p_{\text {нц }}-\right.$ дипольный момент НЦ-иона; предполагаем $p \approx 1$ еА̊). В связи с тем, что в ряду $\mathrm{I}^{-} \rightarrow \mathrm{Br}^{-} \rightarrow$ $\rightarrow \mathrm{Cl}-\Delta$ растет, влияние электрического поля в $\mathrm{KCl}-\mathrm{In}$ и $\mathrm{KBr}-\mathrm{In}$ было выражено еще слабее, чем в KI-In.

Следует отметить, что хотя исследования свойств кристаллов с НЦ-примесными нонами проводятся уже более 20 лет (см., напр., обзоры $\left.\left[{ }^{7,11}\right]\right)$, проявления НЦ-смещения примеси в возбужденном электронном состоянии наблюдались только для $\mathrm{Cu}^{+}$-центров в некоторых ЩГК $\left[{ }^{12-14}\right]$. Очень интересной особенностью исследуемых систем является то, что НЦ-смещение иона примеси в РВС наблюдается в них наряду с сильным ЭЯТ в этом состоянии.

Авторы выражают глубокую благодарность В. Хижнякову за полезное обсуждение результатов работы и Г. Завту за предоставление программы и помощь при расчетах на ЭВМ.

\section{Л И Т Е РА Т У Р А}

1. Зазубович С. Г., Нагирный В. П., Соовик T. А. Оптика и спектр., 62, вып. 6, 1294-1299 (1987).

2. Nagirnyi, V., Soovik, T., Zazubovich, S. Phys. status solidi (b), 126, 653-658 (1984).

3. Li, M. S., de Souza, M., Lüty, F. Phys. Rev. B, 7, № 10, 4677-4682 (1973).

4. Li, M. S., de Souza, M. Phys. status solidi (b), 92, № 1, 287-291 (1979).

5. Ребане К., Сильд О. Изв. АН ЭССР. Физ. Матем., 19, № 3, 311-319 (1970).

6. Берсукер И. Б., Полингер В. З. Вибронные взаимодействия в молекулах и кристаллах. М., «Наука», 1983.

7. Glinchuk, M. D. In: The Dynamical Jahn-Teller Effect in Localized Systems (eds Yu. E. Perlin, M. Wagner). Elsevier Sci. Publishers B. V., North-Holland, 1984, $819-872$.

8. Hizhnyakov, V., Kristoffel, N. In: The Dynamical Jahn-Teller Effect in Localized Systems (eds Yu. E. Perlin, M. Wagner). Elsevier Sci. Publishers B. V., North-Holland, 1984, 385-438.

9. Romestain, R., Le Si Dang, Merle d'Aubigne, Y., Fukuda, A. Semicond. and Insulators, 3, 175-193 (1978).

10. Романов Н. Г., Вещунов Ю. П., Ветров В. А., Баранов П. Г. ФТТ, 23, вып. 10, $2900-2908$ (1981).

11. Lüty, F. Suppl. J. de Phys., 34, C9-49-C9-59 (1973).

12. Piccirilli, M., Spinolo, G. Phys. Rev. B, 4, № 4, 1339-1343 (1971).

13. Mack, S. A., Van Sciver, W. J. Phys. status solidi (b), 46, 193-201 (1971).

14. Keich, D. I., Bieg, K. W., Drickamer, H. G. Phys. Rev. B, 16, № 10, 4599-4607 (1977).
Институт физики
Академии наук Эстонской ССР
Поступила в редакцию 24/II 1987

\section{Svetlana ZAZUBOVITS, V. NAGIRNOI, T. SOOVIK}

\section{UUS MITTETSENTRAALNE SUSTEEM - ELAVHOBEDASARNASED IOONID LEELISHALOGENIIDKRISTALLIDES}

On uuritud leelishalogeniidkristallides paiknevate In+-tsentrite tripletse luminestsentsi kustumise kineetikat ja elektrivälja mõju sellele madalatel temperatuuridel. On näidatud, et kaaliumi ja rubiidiumi halogeniidides nihkub $\mathrm{In}^{+}$relakseerunud ergastatud seisundis (RES) kristallvōre sōlmest suunas, mis on risti Jahn-Telleri moonutuse teljega. In ${ }^{+}$-iooni erinevate mittetsentraalsete asendite vahel toimuvad tunnelüleminekud pōhjustavad tripletse RES-i adiabaatilise potentsiaali tetragonaalsete metastabiilsete miinimumide löhenemist. Tunnelnivoode kiirgusliku lagunemisega seonduvad kaks aeglast kustumiskomponenti, mis ilmnevad nende süsteemide $A_{T}$-kiirguses madalatel temperatuuridel. Saadud andmete baasil on interpreteeritud $\mathrm{Ga}^{+}$-tsentrite RES-i ortorombiline struktuur, mis on varem kindlaks tehtud elektronide paramagnetilise resonantsi optilise detekteerimise meetodil, 


\section{NEW OFF-CENTRE SYSTEM: MERCURY-LIKE IONS IN ALKALI HALIDES}

In the previous paper ${ }^{[1}$ ] we discussed two slow decay components found in lowtemperature (below $4 \mathrm{~K}$ ) decay kinetics of the $A_{T}$ emission on $\mathrm{In}^{+}$centres in potassium and rubidium halides. The decay times of these components differ not more than 2.8 times and their light sums are comparable. The emission and excitation spectra of the components practically coincide and their polarization spectra are similar. Both components are polarized along the $C_{4}$ axis of the crystal. In [ [ $\left.{ }^{1}\right]$ the two slow decay components of the $A_{T}$ emission were connected with off-centre displacement of $\mathrm{In}^{+}$ion in the $A_{T}$ relaxed excited state (RES). In $\mathrm{NaCl}$ : In only one slow component exists.

This paper is an account of a careful study of the temperature dependencies of the decay times and light sums of the components. The influence of the electric field $\vec{B}$

$(E \|[100])$ on the decay kinetics of the polarized $A_{T}$ emission has been investigated at about 2.6 and $1.8 \mathrm{~K}$. It is shown that the off-centre displacement of $\mathrm{In}^{+}$ion in the triplet RES occurs in one of the $\langle 100\rangle$ directions perpendicular to the Jahn-Teller distortion axis. Quick tunnel transitions between various off-centre positions lead to the tunnel splitting of tetragonal metastable minima of triplet RES. The observed two slow decay components of the $A_{T}$ emission are connected with the radiation decay of two tunnel states.

The orthorhombic structure of the $A_{T}$ RES observed several years ago $\left[{ }^{9,10}\right]$ in $\mathrm{Ga}^{+}$ centres in some alkali halides by using the technique of the optical detection of electron paramagnetic resonance is explained here as the evidence of an analogous off-centre displacement of $\mathrm{Ga}^{+}$ion in RES, 